\title{
The spectrum of hearing abnormalities in patients living with diabetes mellitus
}

\author{
S Pillay, ${ }^{1}$ MB ChB, FCP (SA), MMed (Int Med), PhD; K H Naidoo, ${ }^{2}$ MBBS; K Msimang, ${ }^{3}$ BSc (Speech and Hearing Therapy) \\ ${ }^{1}$ Department of Internal Medicine, King Edward VIII Hospital and School of Clinical Medicine, University of KwaZulu-Natal, Durban, South Africa \\ ${ }^{2}$ Diabetes Clinic, Edendale Hospital, Pietermaritzburg, South Africa \\ ${ }^{3}$ Department of Audiology, Edendale Hospital, Pietermaritzburg, South Africa
}

Corresponding author: S Pillay (drspillay@iafrica.com)

\begin{abstract}
Background. Diabetes mellitus (DM) causes both micro- and macrovascular complications. The cochlea and auditory nerves are therefore at increased risk from DM-related complications due to microangiopathy, neuropathy or mitochondrial damage. Limited data are available from Africa detailing the association between DM and hearing impairment (HI).

Objectives. To describe the prevalence and spectrum of and associations with HI in patients living with DM (PLWD) with and without HIV infection.

Methods. This was an analytical cross-sectional study conducted between 1 October and 31 December 2019 at the Edendale Hospital diabetes and audiology clinics in Pietermaritzburg, South Africa. PLWD had an audiological assessment using pure-tone audiometry together with a questionnaire enquiring about tinnitus, vertigo, dizziness and HI.

Results. A total of 296 PLWD (89.2\% with type 2 DM) were enrolled, of whom 154 (52.0\%) had HI. Type 2 PLWD had a significantly higher prevalence of reported symptoms and confirmed audiological HI, which was most often bilateral. The most frequent HI categories were mild to moderate, mild, and moderate to severe $(33.8 \%$ v. $25.9 \%$ v. $23.9 \%$, respectively). Significant associations were noted between dizziness $(p=0.045)$, reported hearing loss $(p<0.001)$ and objective evidence of HI (all categories except mild). HI was significantly associated with age $>50$ years, DM duration $\leq 10$ years, female gender, hypertension, increased low-density lipoprotein and total cholesterol (TC), lower high-density lipoprotein cholesterol, suboptimal glycaemic control, non-proliferative retinopathy, sensory neuropathy and obesity ( $p<0.05$ ). Approximately half (48.9\%) of PLWD and HIV infection had HI, and they were significantly younger and had higher TC levels than PLWD without HIV $(p<0.05)$.

Conclusions. We showed that HI occurs in over half of PLWD, usually within the first 10 years after diagnosis of DM. Symptoms of HI were shown to have positive associations with all HI categories except mild. A high level of vigilance for HI must be maintained in PLWD.

S Afr Med J 2021;111(10):1006-1017. https://doi.org/10.7196/SAMJ.2021.v111i10.15863
\end{abstract}

Hearing impairment (HI) is a significant disability, affecting 278 million people globally. ${ }^{[1]}$ The burden inflicted by HI, like those of diabetes mellitus (DM) and hypertension (HPT), is felt to the greatest extent in low- to middle-income countries. ${ }^{[1]}$

$\mathrm{DM}$ is a metabolic disorder capable of causing both micro- and macrovascular complications. The cochlea and auditory nerves are therefore at risk from these DM-related complications, in the form of microangiopathy, neuropathy or mitochondrial damage. ${ }^{[2,3]}$ Although HI has been observed for decades in people living with diabetes (PLWD), there is still considerable debate as to whether HI and DM are related. Globally, studies have shown that the prevalence of $\mathrm{HI}$ in PLWD ranges from $13 \%$ to $95 \% .{ }^{[4-7]}$ This relationship has been documented in patients with both types 1 and 2 DM. $^{[5,6,8,9]}$ In contrast, studies by Harner, ${ }^{[10]}$ Schuknecht ${ }^{[11]}$ and Bainbridge et al..$^{[12]}$ among others, have shown that there is no association between DM and HI.

When present, $\mathrm{HI}$ in PLWD is usually bilateral, ${ }^{[13-16]}$ and symptoms can range from tinnitus to dizziness, subjective $\mathrm{HI}$ and vertigo, while some patients are asymptomatic. ${ }^{[17-19]}$ Poor glycaemic control is associated with increased risks of DM-related complications. ${ }^{[20]}$ $\mathrm{HI}$ as a complication would appear to be no different. However, the relationship between glycaemic control in PLWD and HI remains controversial. Lerman-Garber et al. ${ }^{[14]}$ and Agrawal et al. ${ }^{[15]}$ found positive correlations between poorly controlled DM and HI. In contrast, Ashkezari et al., ${ }^{[21]}$ Bainbridge et al. ${ }^{[12]}$ and Kakarlapudi et al. ${ }^{[4]}$ found no such association.

Results of studies performed globally have been conflicting with regard to $\mathrm{HI}$ and duration of $\mathrm{DM},{ }^{[7,13,17,21]}$ gender, ${ }^{[5,13,22,23]}$ patient age $^{[13,24,25]}$ and type of antidiabetic therapy. ${ }^{[4,12,26-28]}$ Similarly, findings with regard to the relationship between DM-related complications and HI have also been inconclusive, with some studies demonstrating a positive relationship between DM-related neuropathy ${ }^{[12,22]}$ and retinopathy ${ }^{[21]}$ and HI, while others showed none. ${ }^{[14]}$ Bainbridge et al. ${ }^{[12]}$ showed that patients with HI had an increased prevalence of albuminuria, while Shen and Hsieh ${ }^{[29]}$ demonstrated that HI was linked to serum creatinine levels and inversely related to glomerular filtration rate (GFR).

Obesity remains a major hurdle in the optimal management of DM. Both Curhan et al. ${ }^{[30]}$ and Fransen et al. ${ }^{[31]}$ demonstrated a positive correlation between body mass index (BMI) and HI. Hyperlipidaemia is a common comorbidity in PLWD and is a risk factor for cardiovascular morbidity and mortality. Swaminathan et al. ${ }^{[32]}$ showed that increased total cholesterol (TC), increased triglycerides and increased low-density lipoprotein cholesterol (LDLC) can each increase the risk of HI in PLWD. Evans et al. ${ }^{[33]}$ and Dabrowski et al. ${ }^{[24]}$ demonstrated the same association between triglyceride level and HI. High-density lipoprotein cholesterol (HDLC) is known to have cardioprotective properties. ${ }^{[34]}$ Suzuki et al., ${ }^{[35]}$ 
Bainbridge et al. ${ }^{[12]}$ and Dabrowski et al. ${ }^{[24]}$ have found that low levels of HDL-C are associated with an increased risk of HI.

Limited data relating to DM and $\mathrm{HI}$ are available from Africa and South Africa (SA). In their study conducted in Nigeria, a country similar to SA in terms of its economy, Idugboe et al. ${ }^{[28]}$ showed that HI was common in PLWD (71.4\%) and that the HI was not associated with gender, duration of DM or glycaemic control. They showed that HI occurred earlier in Nigerian patients compared with other studies such as the Wisconsin ${ }^{[36]}$ and Blue Mountains ${ }^{[37]}$ studies, conducted in the USA and Australia, respectively. Most of the PLWD in Idugboe et al.'s ${ }^{[28]}$ study had mild HI, followed by moderate and severe categories $(44.3 \%, 21.4 \%$ and $5.7 \%$, respectively), and were $<50$ years of age; however, it must be noted that their study only included patients between 31 and 64 years of age, and excluded patients with HPT and/or chronic kidney disease.

The only other African study describing HI in PLWD was done in SA by Hlayisi et al., ${ }^{[38]}$ who found that HI was increased in PLWD (55\%), was common in patients $<49$ years of age, was more prevalent in males than in females, and had a positive correlation with duration of DM. However, they found no link between glycated haemoglobin (HbAlc) and $\mathrm{HI}$.

\section{Objectives}

Having accepted that the majority of studies have demonstrated that there is an association between DM and HI, our objectives were first to describe the spectrum of HI in PLWD and then to determine whether any of the associations mentioned above between $\mathrm{HI}$ and PLWD were validated in patients visiting a diabetes clinic at a regional hospital in Pietermaritzburg, KwaZulu-Natal Province. Interestingly, none of the studies mentioned earlier, which were done in both developed and developing countries, assessed whether there was any association between HI and HIV infection in PLWD. This correlation was also searched for in our study.

\section{Methods}

This was an analytical cross-sectional study conducted between 1 October and 31 December 2019 at the Edendale Hospital diabetes and audiology clinics.

All patients aged $>18$ years visiting the diabetes clinic were informed about the study, and those who consented to take part completed informed consent forms. In addition to their routine comprehensive diabetes management, these patients were also directed to the audiology clinic, where an appointment for a pure-tone audiometry (PTA) test was made. At the audiology assessment, the audiologist presented a simple patient symptom questionnaire (tinnitus, vertigo, dizziness and $\mathrm{HI}$ ) before performing PTA. PTA testing was performed by trained audiometric technicians using a GSI 67 audiometer (GrasonStadler Inc., USA) equipped with TDH-39 supra-aural earphones (Telephonics Co., USA) in a dedicated sound-attenuating booth. For quality control and quality assurance, the audiometers are calibrated annually in accordance with the standards of the American National Standards Institute (ISO 389:1991), and biological calibration checks were performed daily prior to the first examination. The ambient noise level was monitored with a sound-level meter.

Pure-tone air conduction thresholds were measured in $\mathrm{dB}$ hearing level (dBHL) for both ears at $0.5,1.0$ and $2.0 \mathrm{kHz}$ and averaged for each ear. Hearing loss was defined as a pure-tone average of thresholds at $0.5,1.0$ and $2.0 \mathrm{kHz}$ of $>25 \mathrm{~dB}$ in both right and left ears. Like Bener et al. ${ }^{[39]}$ we defined the spectrum of $\mathrm{HI}$ in our study as: (i) mild 26 - $40 \mathrm{dBHL}$; (ii) mild to moderate - $41-55 \mathrm{dBHL}$; (iii) moderate to severe - 56 - $70 \mathrm{dBHL}$; $(\mathrm{iv})$ severe - 71 - $90 \mathrm{dBHL}$; and $(v)$ profound $91-120 \mathrm{dBHL}$.
Optimal glycaemic control was defined as in the SA diabetes guidelines as $\mathrm{HbAlc} \leq 7 \% .{ }^{[40]} \mathrm{HbAlc}$ levels were determined using a National Glycohaemoglobin Standardisation Programme-accredited turbidimetric inhibition immunoassay for haemolysed whole blood on a Siemens Dimension EXL 200 apparatus (Siemens Healthcare Diagnostics Inc., Germany).

Height, weight and sitting blood pressure were measured by trained nurses. BMI was calculated as $\mathrm{kg} / \mathrm{m}^{2}$. GFRs were used to stratify renal impairment (Kidney Disease: Improving Global Outcomes (KDIGO) 2012 chronic kidney disease criteria). ${ }^{[41]}$

Fundoscopy was performed by the Edendale Hospital ophthalmology clinic, and retinopathy was classified as nonproliferative or proliferative. The diagnosis of peripheral sensory neuropathy was made from both a history of paraesthesiae and clinical examination.

The Edendale Hospital diabetes data sheet was used for data collection. This has been approved by the University of KwaZuluNatal Biomedical Research and Ethics Committee (UKZN BREC) (ref. no. BCA 194/15). Ethics approval for this study was obtained from the UKZN BREC (ref. no. BE 250/19).

\section{Results}

\section{Demographics}

A total of 296 PLWD were enrolled in the study, with the majority (89.2\%) having type 2 DM (T2DM) ( $n=264$ v. 32, respectively; $p<0.001)$.

Table 1 describes the differences noted when the overall cohort was subdivided according to type of DM. Compared with patients with type 1 DM (T1DM), those with T2DM were significantly older, had had DM for longer and had a higher prevalence of HPT, and T2DM was more prevalent in females. HIV infection was significantly more common in patients with T1DM. Glycaemic control was suboptimal in both types of DM, patients with T1DM having significantly poorer control. Over half of the patients with T2DM (51.1\%) were obese. Patients with T2DM had a substantially higher prevalence of diabetes-related complications (non-proliferative retinopathy, sensory neuropathy, and renal impairment with GFR $<60 \mathrm{~mL}$ / $\min / 1.73 \mathrm{~m}^{2}$ ) and symptoms of dizziness, vertigo, tinnitus and subjective HI than those with T1DM $(p<0.001)$.

A significant proportion of patients in the entire cohort had suboptimal glycaemic control $(n=233 / 296 ; 78.7 \%)$. This was particularly evident in female patients with T2DM $(p<0.001)$. Patients with comorbidities in the form of HPT, obesity and HIV infection and those on antiretroviral therapy (ART) had significantly poorer glycaemic control than those without comorbidities $(p<0.05)$. Diabetes-related complications in the form of sensory neuropathy, non-proliferative and proliferative retinopathy, and renal impairment were more common in patients with $\mathrm{HbA} 1 \mathrm{c}>7 \%(p<0.05)$. Reported symptoms of vertigo and tinnitus were more prevalent in patients with poor glycaemic control $(p<0.001)$. Confirmed HI was considerably more common in patients with $\mathrm{HbAlc}>7 \%(p<0.001)$, especially in the mild to moderate and moderate to severe categories. Bilateral ear involvement was more common in patients with suboptimal glycaemic control $(p<0.001)$. A significant number of patients aged $>50$ years, with a BMI $>25$ and with suboptimal lipid control had relatively poor glycaemic control $(p<0.001)$.

\section{Analysis of patients with $\mathrm{HI}$}

A total of 154 PLWD (52.0\%) had confirmed HI, with 201 individual ears being identified as affected.

The categories of HI found (in descending order of frequency) were mild to moderate, mild, moderate to severe, severe and 
Table 1. Subdivision of patient population according to type of DM $(N=296)$

\begin{tabular}{|c|c|c|c|}
\hline & Type 1 DM $(N=32 ; 10.8 \%)$ & Type 2 DM $(N=264 ; 89.2 \%)$ & $p$-value \\
\hline Age (years), median (IQR) & $29.5(22.5-34)$ & $59(61-67)$ & $<0.001$ \\
\hline \multicolumn{4}{|l|}{ Gender, $n(\%)$} \\
\hline Male & $13(40.6)$ & $54(20.5)$ & $<0.001$ \\
\hline Female & $19(59.4)$ & $210(79.5)$ & $<0.001$ \\
\hline Duration of DM (years), median (IQR) & $6.5(3-12)$ & $9(4-17)$ & 0.049 \\
\hline Patients with HPT, $n(\%)$ & $10(31.3)$ & $243(92.1)$ & $<0.001$ \\
\hline HIV-infected patients, $n(\%)$ & $9(28.1)$ & $36(13.6)$ & $<0.001$ \\
\hline Patients on ART, $n(\%)$ & $8(25.0)$ & $30(11.4)$ & $<0.001$ \\
\hline \multicolumn{4}{|l|}{ Test results, median (IQR) } \\
\hline $\operatorname{HbA1c}(\%)$ & $10.6(8.96-11.35)$ & $9.2(7.4-10.9)$ & 0.02 \\
\hline $\mathrm{TC}(\mathrm{mmol} / \mathrm{L})$ & $4.3(3.5-5.5)$ & $4.4(3.6-5.1)$ & 0.668 \\
\hline Triglycerides (mmol/L) & $0.98(0.83-1.61)$ & $1.48(1.03-1.46)$ & 0.006 \\
\hline HDL-C (mmol/L) & $1.19(1.04-1.75)$ & $1.22(1.03-1.46)$ & 0.375 \\
\hline LDL-C (mmol/L) & $2.68(1.85-3.79)$ & $2.3(1.69-3.06)$ & 0.147 \\
\hline CD4 count (cells $/ \mu \mathrm{L})$ & $559(491-913)$ & $600(409.25-954.5)$ & 0.745 \\
\hline Creatinine $(\mathrm{mmol} / \mathrm{L})$ & $62(55.25-75.24)$ & $83(66-119)$ & $<0.001$ \\
\hline $\operatorname{BMI}\left(\mathrm{kg} / \mathrm{m}^{2}\right)$ & $26(22.75-30.25)$ & $32(28-38)$ & $<0.001$ \\
\hline Urinary PCR & $0.01(0.01-0.123)$ & $0.03(0.018-0.123)$ & 0.475 \\
\hline \multicolumn{4}{|l|}{ Diabetes-related complications, $n(\%)$} \\
\hline Non-proliferative retinopathy & $3(9.4)$ & $25(9.5)$ & $<0.001$ \\
\hline Proliferative retinopathy & 0 & $8(3.0)$ & - \\
\hline Sensory neuropathy & $9(28.1)$ & $127(48.1)$ & $<0.001$ \\
\hline \multicolumn{4}{|l|}{ Antidiabetic therapy, $n(\%)$} \\
\hline Metformin only & 0 & $17(6.4)$ & - \\
\hline Insulin only & $27(84.4)$ & $134(50.8)$ & $<0.001$ \\
\hline OADs plus insulin & $5(15.6)$ & $111(42.0)$ & $<0.001$ \\
\hline \multicolumn{4}{|l|}{ Patient characteristics, $n(\%)$} \\
\hline \multicolumn{4}{|l|}{ Age (years) } \\
\hline$\leq 50$ & $31(96.9)$ & $59(22.4)$ & 0.003 \\
\hline$>50$ & $1(3.1)$ & $205(77.7)$ & $<0.001$ \\
\hline \multicolumn{4}{|l|}{ Duration of DM (years) } \\
\hline$\leq 10$ & $23(71.9)$ & $138(52.3)$ & $<0.001$ \\
\hline$>10$ & $9(28.1)$ & $111(42.1)$ & $<0.001$ \\
\hline \multicolumn{4}{|l|}{$\mathrm{BMI}^{\star}\left(\mathrm{kg} / \mathrm{m}^{2}\right)$} \\
\hline$\leq 25$ & $13(40.6)$ & $39(14.8)$ & $<0.001$ \\
\hline$>25$ & $16(5)$ & $201(76.1)$ & $<0.001$ \\
\hline \multicolumn{4}{|l|}{ HbAlc (\%) } \\
\hline$\leq 7$ & $2(6.3)$ & $49(18.6)$ & $<0.001$ \\
\hline$>7$ & $30(93.8)$ & $203(76.9)$ & $<0.001$ \\
\hline \multicolumn{4}{|l|}{$\mathrm{TC}^{\star}(\mathrm{mmol} / \mathrm{L})$} \\
\hline$\leq 4$ & $12(37.5)$ & $95(36.0)$ & $<0.001$ \\
\hline$>4$ & $19(59.4)$ & $153(58.0)$ & $<0.001$ \\
\hline \multicolumn{4}{|l|}{ Triglyceride $^{*}(\mathrm{mmol} / \mathrm{L})$} \\
\hline$\leq 1.7$ & $24(75.0)$ & $149(56.4)$ & $<0.001$ \\
\hline$>1.7$ & 7 (21.9) & $94(35.6)$ & $<0.001$ \\
\hline \multicolumn{4}{|l|}{ 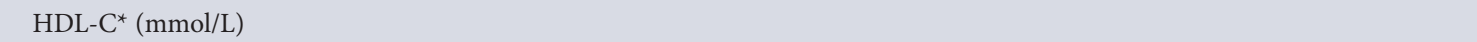 } \\
\hline$\leq 1.2$ & $14(43.8)$ & $96(36.4)$ & $<0.001$ \\
\hline$>1.2$ & $13(40.6)$ & $109(41.3)$ & $<0.001$ \\
\hline \multicolumn{4}{|l|}{$\mathrm{LDL}^{-\mathrm{C}^{\star}}(\mathrm{mmol} / \mathrm{L})$} \\
\hline$\leq 1.8$ & $4(12.5)$ & $54(20.5)$ & $<0.001$ \\
\hline$>1.8$ & $20(62.5)$ & $105(39.8)$ & $<0.001$ \\
\hline \multicolumn{4}{|l|}{ Creatinine $^{\star}(\mathrm{mmol} / \mathrm{L})$} \\
\hline$\leq 104$ & $29(90.6)$ & $169(64.0)$ & $<0.001$ \\
\hline$>104$ & $2(6.3)$ & 79 (29.9) & $<0.001$ \\
\hline
\end{tabular}


Table 1. (continued) Subdivision of patient population according to type of DM ( $N=296)$

\begin{tabular}{|c|c|c|c|}
\hline & Type 1 DM $(N=32 ; 10.8 \%)$ & Type 2 DM $(N=264 ; 89.2 \%)$ & $p$-value \\
\hline \multicolumn{4}{|l|}{$\mathrm{GFR}^{*}\left(\mathrm{~mL} / \mathrm{min} / 1.73 \mathrm{~m}^{2}\right)$} \\
\hline$<60$ & $2(6.3)$ & $108(40.9)$ & $<0.001$ \\
\hline$\geq 60$ & $29(90.6)$ & $140(53.0)$ & $<0.001$ \\
\hline \multicolumn{4}{|l|}{ Symptoms reported, $n$ (\%) } \\
\hline Dizziness & $8(25.0)$ & $112(42.4)$ & $<0.001$ \\
\hline Vertigo & $5(15.6)$ & $59(22.4)$ & $<0.001$ \\
\hline Tinnitus & $9(28.1)$ & $119(45.1)$ & $<0.001$ \\
\hline Subjective HI & $4(12.5)$ & $118(44.7)$ & $<0.001$ \\
\hline \multicolumn{4}{|c|}{ Confirmed hearing loss, $n(\%)$} \\
\hline Right ear & $4(12.5)$ & $131(49.6)$ & $<0.001$ \\
\hline Left ear & $4(12.5)$ & $136(51.5)$ & $<0.001$ \\
\hline Bilateral involvement & $3(9.4)$ & $73(27.7)$ & $<0.001$ \\
\hline
\end{tabular}

profound $(33.8 \%$ v. $25.9 \%$ v. $23.9 \%$ v. $11.4 \%$ v. $5.0 \%$, respectively). Aproximately half $(n=76 / 154 ; 49.4 \%)$ of PLWD with HI had bilateral ear involvement across all categories of $\mathrm{HI}(p<0.001)$. Patients with T2DM had a significantly higher prevalence of HI (both unilateral and bilateral; $p<0.001)$ in all HI categories.

Table 2 demonstrates that the cohort of patients with confirmed HI were significantly older than those without HI, had had DM for longer, had a higher prevalence of concomitant HPT and sensory neuropathy, higher creatinine levels and lower GFRs, mostly had T2DM, and had a higher prevalence of dizziness and self-reported hearing loss.

The majority of PLWD aged $>60$ years had $\mathrm{HI}(n=82$ v. $26 ; p<0.001)$. In the $\mathrm{HI}$ cohort, bilateral ear involvement was significantly more common than unilateral disease $(p<0.001)$. No significant difference $(p<0.05)$ was noted with regard to the side of ear involvement.

Table 3 shows that the prevalences of HPT and sensory neuropathy were significantly higher in the severe and profound HI categories. The majority of patients with HI were aged $>40$ years (93.7\%), with $53.1 \%$ of these patients being $>60$ years old. The mild to moderate HI category was more common in patients aged $41-60$ years, usually with a duration of $\mathrm{DM} \leq 10$ years, while mild to moderate and moderate to severe categories were most common in patients aged $>60$ years. A significant proportion of patients across all $\mathrm{HI}$ categories had a BMI $>25(p<0.05)$. TC was significantly elevated in patients in the mild and moderate to severe categories, while LDL-C was elevated in the mild HI category $(p<0.05)$. Glycaemic control was suboptimal across all $\mathrm{HI}$ categories $(p=0.011)$. Approximately half of the patients with confirmed HI had subjective symptoms of dizziness, tinnitus and hearing loss, and just over a quarter reported vertigo.

\section{Subdivision of patient cohort based on HIV status}

Approximately half of PLWD with HIV infection had evidence of HI ( $n=22 / 45 ; 48.9 \%)$. Table 4 shows that mild and severe categories of $\mathrm{HI}$ were significantly more common in HIV-infected PLWD compared with their HIV-uninfected counterparts. These PLWD and HIV with HI were significantly younger $(p<0.001)$ and had higher TC levels $(p=0.044)$ compared with PLWD without HIV infection.

\section{Symptoms of HI}

Vertigo and tinnitus occurred more frequently in patients aged 41 - 60 years, while tinnitus and subjective hearing loss were more common in those aged $>60$ years. Table 5 shows that PLWD with suboptimal glycaemic control, those with a BMI $>25$ and female patients with
T2DM had a significantly higher prevalence of all four symptoms.

The majority of patients with HI had HPT as a comorbidity, while over half of the patients with HI who reported any of the four symptoms also had elevated TC and/or LDL-C levels.

Mild to moderate HI was mostly associated with symptoms of vertigo $(p=0.019)$ and subjective hearing loss $(p=0.035)$. Moderate to severe HI was associated with dizziness $(p=0.024)$, vertigo ( $p=0.036)$ and subjective hearing loss $(p<0.001)$, while both severe and profound HI were significantly related to subjective hearing loss ( $p=0.007$ and $p=0.018$, respectively). No significant association was noted between the mild category and any of the four symptoms, which shows that this stage of $\mathrm{HI}$ is easy to miss without proper audiological screening.

All four symptoms were significantly associated with female gender. Dizziness, tinnitus and subjective hearing loss were associated with T2DM $(p<0.05)$, while subjective hearing loss was strongly associated with HPT $(p=0.004)$, a lower GFR $(p=0.004)$, sensory neuropathy $(p=0.001)$ and total HI $(p<0.001)$ (Table 5).

In univariate analysis, the unadjusted odds ratio revealed that $\mathrm{HI}$ was significantly associated with T2DM, HPT, sensory neuropathy, duration of DM $\leq 10$ years, age $>50$ years, a lower GFR and elevated creatinine levels. No significant relationship was found between HIV status, ART use, weight and HI. A significant association was found between symptoms (dizziness, vertigo, subjective hearing loss) and objective HI (Table 6).

After adjusting for age and gender, age $>50$ years and lower HDL-C were significantly associated with HI. Patients with HPT and elevated LDL-C were at 4.22 and 3.81 times greater risk of developing HI, respectively, while patients who had poorer $\mathrm{HbAlc}$, non-proliferative retinopathy and sensory neuropathy were at 2.03 v. 1.35 v. 1.3 times greater risk of developing HI (Table 7).

\section{Discussion}

$\mathrm{DM}$ is a multisystem disease that is capable of inflicting damage to the auditory nerve through its micro- and macrovascular complications. However, there is a dearth of literature from Africa on the association between DM and $\mathrm{HI}$ and the need for audiological screening in routine diabetes care.

The bulk of our patient cohort were female, of older age, had comorbidities in the form of T2DM, HPT and obesity, and had diabetes-related complications (non-proliferative retinopathy, peripheral sensory neuropathy, and renal impairment as determined by higher creatinine levels and a lower GFR). 


\section{Table 2. Differences between the cohorts with and without HI $(N=296)$}

\begin{tabular}{|c|c|c|c|}
\hline & No HI $(N=142 ; 48.0 \%)$ & HI $(N=154 ; 52.0 \%)$ & $p$-value \\
\hline Age (years), median (IQR) & $50(38-59)$ & $62.5(55-70)$ & $<0.001$ \\
\hline \multicolumn{4}{|l|}{ Gender, $n(\%)$} \\
\hline Male & $34(23.9)$ & $33(21.4)$ & 0.903 \\
\hline Female & $108(76.1)$ & $121(78.6)$ & 0.390 \\
\hline Male v. female & $p<0.001$ & $p<0.001$ & \\
\hline \multicolumn{4}{|l|}{ Type of DM, $n(\%)$} \\
\hline T1DM & $27(19.0)$ & $5(3.2)$ & $<0.001$ \\
\hline $\mathrm{T} 2 \mathrm{DM}$ & $115(81.0)$ & $149(96.8)$ & $<0.001$ \\
\hline Duration of DM (years), median (IQR) & $7(3-13)$ & $11(5-18)$ & $<0.001$ \\
\hline Patients with HPT, $n(\%)$ & $107(75.4)$ & $146(94.8)$ & 0.014 \\
\hline HIV-infected patients, $n$ (\%) & $23(16.2)$ & $22(14.3)$ & 0.746 \\
\hline Patients on ART, $n(\%)$ & $17(12.0)$ & $21(13.6)$ & 0.516 \\
\hline \multicolumn{4}{|l|}{ Test results, median (IQR) } \\
\hline HbAlc (\%) & $9.8(7.53-11.1)$ & $9.1(7.5-10.95)$ & 0.312 \\
\hline $\mathrm{TC}(\mathrm{mmol} / \mathrm{L})$ & $4.4(3.5-5.2)$ & $4.4(3.6-5.2)$ & 0.637 \\
\hline Triglyceride $(\mathrm{mmol} / \mathrm{L})$ & $1.41(0.95-1.93)$ & $1.5(0.1-2.36)$ & 0.116 \\
\hline HDL-C (mmol/L) & $1.17(1.01-1.52)$ & $1.24(1.06-0.48)$ & 0.499 \\
\hline LDL-C (mmol/L) & $2.36(1.78-3.12)$ & $2.39(1.66-3.18)$ & 0.696 \\
\hline CD4 count (cells/ $\mu \mathrm{L})$ & $678(497.75-882.5)$ & $511(405.5-944.5)$ & 0.58 \\
\hline Creatinine $(\mathrm{mmol} / \mathrm{L})$ & $71(59-89.5)$ & $95.5(68-145)$ & $<0.001$ \\
\hline BMI $\left(\mathrm{kg} / \mathrm{m}^{2}\right)$ & $32(26.5-38)$ & $31(28-37)$ & 0.724 \\
\hline Urinary PCR & $0.02(0.01-0.1)$ & $0.03(0.02-0.09)$ & 0.236 \\
\hline \multicolumn{4}{|l|}{ Diabetes-related complications, $n(\%)$} \\
\hline Non-proliferative retinopathy & $11(7.8)$ & $17(11.0)$ & 0.257 \\
\hline Proliferative retinopathy & $3(2.1)$ & $5(3.3)$ & 0.480 \\
\hline Sensory neuropathy & $56(39.4)$ & $80(52.0)$ & 0.04 \\
\hline \multicolumn{4}{|l|}{ Antidiabetic therapy, $n$ (\%) } \\
\hline Metformin only & $7(4.9)$ & $10(6.5)$ & 0.467 \\
\hline Insulin only & $70(49.3)$ & $91(59.1)$ & 0.098 \\
\hline OADs plus insulin & $51(35.9)$ & $47(30.5)$ & 0.686 \\
\hline \multicolumn{4}{|l|}{ Patient characteristics, $n$ (\%) } \\
\hline \multicolumn{4}{|l|}{ Age (years) } \\
\hline$\leq 50$ & $73(51.4)$ & $17(11.0)$ & $<0.001$ \\
\hline$>50$ & $69(48.6)$ & $137(89.0)$ & $<0.001$ \\
\hline \multicolumn{4}{|l|}{ Duration of $\mathrm{DM}^{\star}$ (years) } \\
\hline$\leq 10$ & $90(63.4)$ & $71(46.1)$ & 0.134 \\
\hline$>10$ & $45(31.7)$ & $75(48.7)$ & 0.006 \\
\hline \multicolumn{4}{|l|}{$\mathrm{BMI}^{*}\left(\mathrm{~kg} / \mathrm{m}^{2}\right)$} \\
\hline$\leq 25$ & $27(19.0)$ & $25(16.2)$ & 0.782 \\
\hline$>25$ & $101(71.1)$ & $116(75.3)$ & 0.309 \\
\hline \multicolumn{4}{|l|}{$\operatorname{HbAlc}^{*}(\%)$} \\
\hline$\leq 7$ & $25(17.0)$ & $26(16.9)$ & 0.889 \\
\hline$>7$ & $110(77.5)$ & $123(79.9)$ & 0.394 \\
\hline \multicolumn{4}{|l|}{$\mathrm{TC}^{\star}(\mathrm{mmol} / \mathrm{L})$} \\
\hline$\leq 4$ & $53(37.3)$ & $54(35.1)$ & 0.923 \\
\hline$>4$ & $79(55.6)$ & $93(60.4)$ & 0.286 \\
\hline \multicolumn{4}{|l|}{$\operatorname{Triglyceride}^{*}(\mathrm{mmol} / \mathrm{L})$} \\
\hline$\leq 1.7$ & $87(61.3)$ & $86(55.8)$ & 0.86 \\
\hline$>1.7$ & $42(29.6)$ & $59(38.3)$ & 0.091 \\
\hline \multicolumn{4}{|l|}{ 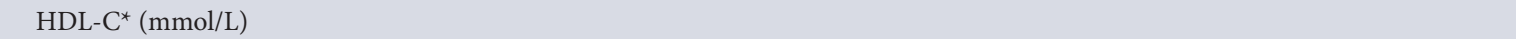 } \\
\hline$\leq 1.2$ & $57(40.1)$ & $53(34.4)$ & 0.703 \\
\hline$>1.2$ & $51(35.9)$ & $71(46.1)$ & 0.070 \\
\hline \multicolumn{4}{|l|}{$\mathrm{LDL}_{-} \mathrm{C}^{*}(\mathrm{mmol} / \mathrm{L})$} \\
\hline$\leq 1.8$ & $28(19.7)$ & $30(19.5)$ & 0.793 \\
\hline$>1.8$ & $62(43.7)$ & $63(40.9)$ & 0.929 \\
\hline
\end{tabular}


Table 2. (continued) Differences between the cohorts with and without $H I(N=296)$

\begin{tabular}{|c|c|c|c|}
\hline & No HI $(N=142 ; 48.0 \%)$ & HI $(N=154 ; 52.0 \%)$ & $p$-value \\
\hline \multicolumn{4}{|c|}{$\mathrm{GFR}^{*}\left(\mathrm{~mL} / \mathrm{min} / 1.73 \mathrm{~m}^{2}\right)$} \\
\hline$<60$ & $28(19.7)$ & $82(53.3)$ & $<0.001$ \\
\hline$\geq 60$ & $105(73.9)$ & $64(41.6)$ & 0.002 \\
\hline \multicolumn{4}{|c|}{ Creatinine $^{\star}(\mathrm{mmol} / \mathrm{L})$} \\
\hline$\leq 104$ & $112(78.9)$ & $86(55.8)$ & 0.065 \\
\hline$>104$ & $21(14.8)$ & $60(39.0)$ & $<0.001$ \\
\hline \multicolumn{4}{|c|}{ Symptoms reported, $n(\%)$} \\
\hline Dizziness & $49(34.5)$ & $71(46.1)$ & 0.045 \\
\hline Vertigo & $25(17.6)$ & $39(25.3)$ & 0.08 \\
\hline Tinnitus & $55(38.7)$ & $73(47.4)$ & 0.112 \\
\hline Subjective HI & $36(25.4)$ & $86(55.8)$ & $<0.001$ \\
\hline
\end{tabular}

Table 3. HI groups stratified by demographic, clinical and biochemical variables $(N=201)$

\begin{tabular}{|c|c|c|c|c|c|c|}
\hline & Mild $(N=52)$ & $\begin{array}{l}\text { Mild to mod. } \\
(N=68)\end{array}$ & $\begin{array}{l}\text { Mod. to severe } \\
(N=48)\end{array}$ & Severe $(N=23)$ & $\begin{array}{l}\text { Profound } \\
(N=10)\end{array}$ & $p$-value \\
\hline Age (years), median (IQR) & $59.5(54.5-67.5)$ & $62.5(56-70.5)$ & $67(60-73)$ & $66.5(59-77)$ & $62.5(55-70)$ & 0.10 \\
\hline \multicolumn{7}{|l|}{ Gender, $n(\%)$} \\
\hline Males $(N=39)$ & $12(23.1)$ & $12(17.7)$ & $7(14.6)$ & $4(17.4)$ & $4(40.0)$ & 0.08 \\
\hline Females $(N=162)$ & $40(76.9)$ & $56(82.3)$ & $41(85.4)$ & $19(82.6)$ & $6(60.0)$ & $<0.001$ \\
\hline \multicolumn{7}{|l|}{ Type of DM, $n(\%)$} \\
\hline T1DM $(N=5)$ & 0 & $3(4.4)$ & $2(4.2)$ & 0 & 0 & 0.09 \\
\hline T2DM $(N=196)$ & $52(100)$ & $65(95.6)$ & $46(95.8)$ & $23(100)$ & $10(100)$ & $<0.001$ \\
\hline Duration of DM (years), median (IQR) & $18(3-18)$ & $12(6-18)$ & $13(7-18)$ & $9(4.75-19)$ & $9(6-13)$ & 0.57 \\
\hline Patients with HPT (N=193), $n(\%)$ & $50(96.2)$ & $64(94.1)$ & $45(93.8)$ & $23(100)$ & $10(100)$ & $<0.001$ \\
\hline HIV-infected patients $(N=29), n(\%)$ & $9(17.3)$ & $10(14.7)$ & $5(10.4)$ & $4(17.4)$ & $1(10.0)$ & 0.050 \\
\hline Patients on ART $(N=27), n(\%)$ & $9(17.3)$ & $10(14.7)$ & $3(6.25)$ & $4(17.4)$ & $1(10.0)$ & 0.023 \\
\hline \multicolumn{7}{|l|}{ Test results, median (IQR) } \\
\hline HbAlc (\%) & $9.3(7.35-11.6)$ & $9.4(8.43-11.48)$ & $8.7(7.55-9.5)$ & $9.4(7.4-10.9)$ & $\begin{array}{l}7.5(6.83- \\
9.05)\end{array}$ & 0.04 \\
\hline $\mathrm{TC}(\mathrm{mmol} / \mathrm{L})$ & $4.4(3.88-4.92)$ & $4.55(3.6-5.4)$ & $4.55(3.6-5.5)$ & $4.35(3.5-5.4)$ & $\begin{array}{l}3.7(3.38- \\
4.88)\end{array}$ & 0.79 \\
\hline Triglyceride $(\mathrm{mmol} / \mathrm{L})$ & $1.33(0.93-2.17)$ & $1.65(1.17-2.52)$ & $1.5(0.99-2.19)$ & $\begin{array}{l}1.49(1.28- \\
2.86)\end{array}$ & $\begin{array}{l}1.05(1.0- \\
2.03)\end{array}$ & 0.88 \\
\hline LDL-C (mmol/L) & $2.41(1.85-3.05)$ & $2.13(1.66-2.99)$ & $2.3(1.66-3.37)$ & $\begin{array}{l}2.11(1.29- \\
3.33)\end{array}$ & $\begin{array}{l}2.03(1.4- \\
2.89)\end{array}$ & 0.90 \\
\hline $\mathrm{HDL}-\mathrm{C}(\mathrm{mmol} / \mathrm{L})$ & $1.23(1.05-1.41)$ & $1.23(1.05-1.52)$ & $\begin{array}{l}1.29(1.07- \\
1.49)\end{array}$ & $\begin{array}{l}1.22(1.11- \\
1.46)\end{array}$ & $\begin{array}{l}1.11(0.96- \\
1.13)\end{array}$ & 0.21 \\
\hline CD4 (cells/ $\mu \mathrm{L})$ & $\begin{array}{l}726.5(413- \\
1061)\end{array}$ & $\begin{array}{l}521(468.02- \\
782.57)\end{array}$ & $\begin{array}{l}936(609- \\
1795.5)\end{array}$ & $\begin{array}{l}182(46.25- \\
312.5)\end{array}$ & $182(182)$ & 0.04 \\
\hline Creatinine (mmol/L) & $78(67-131.63)$ & $91(68.24-133.36)$ & $\begin{array}{l}91(68.25- \\
133.36)\end{array}$ & $94.49(98-151)$ & $\begin{array}{l}101.5(65- \\
144)\end{array}$ & 0.22 \\
\hline $\mathrm{BMI}\left(\mathrm{kg} / \mathrm{m}^{2}\right)$ & $30(26-34.49)$ & $32(28.75-38)$ & $30(28-37.15)$ & $30(27-40)$ & $28.5(28-32)$ & 0.48 \\
\hline Urinary PCR & $0.04(0.01-0.2)$ & $0.03(0.01-0.17)$ & $0.03(0.02-0.06)$ & $0.02(0.05-0.16)$ & $\begin{array}{l}0.06(0.05- \\
0.10)\end{array}$ & 0.50 \\
\hline \multicolumn{7}{|l|}{ Diabetes-related complications, $n(\%)$} \\
\hline Non-proliferative retinopathy $(N=24)$ & $6(11.5)$ & $9(13.2)$ & $7(14.6)$ & $2(8.7)$ & 0 & 0.022 \\
\hline Proliferative retinopathy $(N=5)$ & 0 & $3(4.4)$ & 0 & $2(8.7)$ & 0 & 0.091 \\
\hline Sensory neuropathy $(N=109)$ & $27(51.9)$ & $37(54.4)$ & $23(47.9)$ & $15(65.2)$ & $7(70.0)$ & $<0.001$ \\
\hline \multicolumn{7}{|l|}{ Antidiabetic therapy, $n$} \\
\hline Metformin only & 4 & 6 & 4 & 7 & 0 & $<0.001$ \\
\hline Insulin only & 28 & 41 & 30 & 6 & 6 & $<0.001$ \\
\hline OADs plus insulin & 19 & 19 & 12 & 10 & 4 & 0.004 \\
\hline
\end{tabular}


Table 3. (continued) HI groups stratified by demographic, clinical and biochemical variables $(N=201)$

\begin{tabular}{|c|c|c|c|c|c|c|}
\hline & Mild $(N=52)$ & $\begin{array}{l}\text { Mild to mod. } \\
(N=68)\end{array}$ & $\begin{array}{l}\text { Mod. to severe } \\
(N=48)\end{array}$ & Severe $(N=23)$ & $\begin{array}{l}\text { Profound } \\
(N=10)\end{array}$ & $p$-value \\
\hline \multicolumn{7}{|l|}{ Patient characteristics, $n(\%)$} \\
\hline \multicolumn{7}{|l|}{ Age (years) } \\
\hline$\leq 50$ & $6(11.5)$ & $7(10.3)$ & $4(8.3)$ & $4(17.4)$ & $1(10.0)$ & 0.306 \\
\hline$>50$ & $46(88.5)$ & $61(89.7)$ & $44(91.7)$ & $19(82.6)$ & $9(90.0)$ & $<0.001$ \\
\hline \multicolumn{7}{|l|}{ Duration of $\mathrm{DM}^{*}$ (years) } \\
\hline$\leq 10$ & $27(54)$ & $29(46.8)$ & $19(41.3)$ & $11(52.4)$ & $5(55.6)$ & $<0.001$ \\
\hline$>10$ & $23(46)$ & $33(53.2)$ & $27(58.7)$ & $10(47.6)$ & $4(44.4)$ & $<0.001$ \\
\hline \multicolumn{7}{|l|}{$\mathrm{BMI}^{*}$} \\
\hline$\leq 25$ & $9(18.4)$ & $10(16.4)$ & $6(14)$ & $4(19)$ & $1(10.0)$ & 0.061 \\
\hline$>25$ & $40(81.6)$ & $51(83.6)$ & $37(86)$ & $17(81)$ & $9(90.0)$ & $<0.001$ \\
\hline \multicolumn{7}{|l|}{$\operatorname{HbAlc}^{*}(\%)$} \\
\hline$\leq 7$ & $11(22.4)$ & $6(9)$ & $8(16.7)$ & $4(19)$ & $3(33.3)$ & 0.169 \\
\hline$>7$ & $38(77.6)$ & $61(91)$ & $40(83.3)$ & $17(81)$ & $6(66.7)$ & $<0.001$ \\
\hline \multicolumn{7}{|l|}{$\mathrm{TC}^{*}(\mathrm{mmol} / \mathrm{L})$} \\
\hline$\leq 4$ & $16(32.7)$ & $21(31.8)$ & $17(35.4)$ & $9(40.9)$ & $5(55.6)$ & 0.015 \\
\hline$>4$ & $33(67.3)$ & $45(68.2)$ & $31(64.6)$ & $13(59.1)$ & $4(44.4)$ & $<0.001$ \\
\hline \multicolumn{7}{|l|}{ Triglyceride $^{\star}(\mathrm{mmol} / \mathrm{L})$} \\
\hline$\leq 1.7$ & $30(61.2)$ & $35(53)$ & $32(68.1)$ & $11(55)$ & $5(55.6)$ & $<0.001$ \\
\hline$>1.7$ & $19(38.8)$ & $31(47)$ & $15(31.9)$ & $9(45)$ & $4(44.4)$ & $<0.001$ \\
\hline \multicolumn{7}{|l|}{ 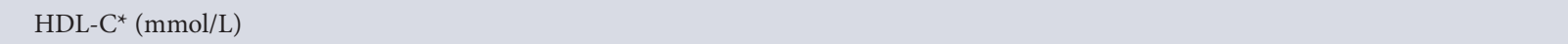 } \\
\hline$\leq 1.2$ & $18(42.9)$ & $22(43.1)$ & $16(38.1)$ & $8(44.4)$ & $7(87.5)$ & 0.018 \\
\hline$>1.2$ & $24(57.1)$ & $29(56.9)$ & $26(61.9)$ & $10(56.6)$ & $1(12.5)$ & $<0.001$ \\
\hline \multicolumn{7}{|l|}{ 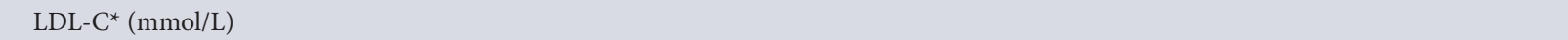 } \\
\hline$\leq 1.8$ & $8(25)$ & $13(38.2)$ & $12(36.4)$ & $6(46.2)$ & $4(50.0)$ & 0.142 \\
\hline$>1.8$ & $24(75)$ & $21(61.8)$ & $21(63.6)$ & $7(53.8)$ & $4(50.0)$ & $<0.001$ \\
\hline \multicolumn{7}{|l|}{$\mathrm{GFR}^{*}\left(\mathrm{~mL} / \mathrm{min} / 1.73 \mathrm{~m}^{2}\right)$} \\
\hline$<60$ & $22(46.8)$ & $35(52.2)$ & $28(60.9)$ & $15(68.2)$ & $7(77.8)$ & $<0.001$ \\
\hline$\geq 60$ & $25(53.2)$ & $32(47.8)$ & $18(39.1)$ & $7(31.8)$ & $2(22.2)$ & $<0.001$ \\
\hline \multicolumn{7}{|l|}{ Creatinine $^{\star}(\mathrm{mmol} / \mathrm{L})$} \\
\hline$\leq 104$ & $30(63.8)$ & $43(64.2)$ & $27(60)$ & $12(54.5)$ & $3(33.3)$ & $<0.001$ \\
\hline$>104$ & $17(36.2)$ & $24(35.8)$ & $18(40)$ & $10(45.5)$ & $6(66.7)$ & 0.010 \\
\hline \multicolumn{7}{|l|}{ Symptoms reported, $n(\%)$} \\
\hline Dizziness $(N=94 ; 47.8 \%)$ & $20(38.5)$ & $31(45.6)$ & $27(56.3)$ & $13(56.5)$ & $3(30.0)$ & $<0.001$ \\
\hline Vertigo $(N=55 ; 27.4 \%)$ & $11(21.2)$ & $22(32.4)$ & $16(33.3)$ & $5(21.7)$ & $1(10.0)$ & $<0.001$ \\
\hline Tinnitus $(N=103 ; 51.2 \%)$ & $23(44.2)$ & $35(51.5)$ & $26(54.2)$ & $12(52.2)$ & $7(70.0)$ & $<0.001$ \\
\hline Hearing loss $(N=118 ; 58.7 \%)$ & $24(46.2)$ & $36(52.9)$ & $34(70.8)$ & $16(69.6)$ & $8(80.0)$ & $<0.001$ \\
\hline
\end{tabular}

A significant majority of patients had suboptimal glycaemic control. Poor control was associated with comorbidities (HPT, obesity and HIV infection) and being on ART. In the cohort of patients with suboptimal glycaemic control, there was a significantly higher prevalence of diabetes-related complications (sensory neuropathy, non-proliferative retinopathy and renal impairment), more reported symptoms of $\mathrm{HI}$ (dizziness, tinnitus, vertigo and subjective $\mathrm{HI}$ ), confirmed HI, bilateral HI and poorer lipid control. Patients with T2DM had a significantly higher prevalence of reported symptoms of $\mathrm{HI}$ and confirmed audiological evidence of hearing loss (both unilateral and bilateral ear involvement).

Our study demonstrated that over half $(52.0 \%)$ of PLWD had evidence of $\mathrm{HI}$ on audiological screening. This is comparable to what Idugboe et al..$^{[28]}$ and Hlayisi et al. ${ }^{[38]}$ showed in their studies conducted in Nigeria and SA, respectively $(52.0 \%$ v. $71.4 \%$ v. $55 \%$, respectively). ${ }^{[28,38]} \mathrm{HI}$ in our study was significantly more common in patients with $\mathrm{T} 2 \mathrm{DM}$, and when present, was usually bilateral. We showed that HI was significantly more prevalent in patients who were female, older, had HPT, had had DM for a longer time, and had evidence of sensory neuropathy.

Although not statistically significant, there was a higher prevalence of HI in PLWD and HIV infection than in their HIV-uninfected counterparts. This association deserves more attention in future studies including a greater number of HIV-infected patients. In our study, PLWD with HIV infection and HI were significantly younger and had higher TC levels than their counterparts. Abnormal lipid metabolism is associated with both HIV infection and/or ART. ${ }^{[42]}$ DM, HIV infection and elevated cholesterol are independent risk factors for cardiovascular disease ${ }^{[20,34,42,43]}$ Our findings suggest a role for audiological screening as part of the routine assessment in both HIV and DM management. Clinicians treating these patients must screen for $\mathrm{HI}$ early in the course of DM, especially in young HIV-infected PLWD.

The top three HI categories in our study were mild to moderate, mild, and moderate to severe, which differed from the results of 


\begin{tabular}{|c|c|c|c|}
\hline & HIV-infected $(N=22)$ & HIV-uninfected $(N=132)$ & $p$-value \\
\hline Total, $n(\%)$ & $22(14.3)$ & $132(85.7)$ & $<0.001$ \\
\hline Age (years), median (IQR) & $52.5(46-57)$ & $65(57-71)$ & $<0.001$ \\
\hline Duration of DM (years), median (IQR) & $8(4.25-13.25)$ & $11(5-19)$ & 0.078 \\
\hline \multicolumn{4}{|l|}{ Test results, median (IQR) } \\
\hline HbAlc (\%) & $9.6(7.7-12.1)$ & $9.1(7.5-10.88)$ & 0.304 \\
\hline $\mathrm{TC}(\mathrm{mmol} / \mathrm{L})$ & $4.75(4.4-5.6)$ & $4.3(3.6-5.13)$ & 0.044 \\
\hline Triglyceride $(\mathrm{mmol} / \mathrm{L})$ & $1.51(1.1-2.2)$ & $1.48(0.9-2.3)$ & 0.647 \\
\hline HDL-C (mmol/L) & $1.32(1.16-1.58)$ & $1.22(1.05-1.47)$ & 0.157 \\
\hline LDL-C (mmol/L) & $2.67(1.95-3.4)$ & $2.39(1.63-3.11)$ & 0.506 \\
\hline Urinary PCR & $0.08(0.03-0.23)$ & $0.03(0.02-0.06)$ & 0.284 \\
\hline Creatinine (mmol/L) & $99(67.24-140.14)$ & $95(65.75-146.23)$ & 0.874 \\
\hline BMI $\left(\mathrm{kg} / \mathrm{m}^{2}\right)$ & $29(25-32)$ & $31.5(28-38)$ & 0.082 \\
\hline \multicolumn{4}{|l|}{ Categories of HI, $n(\%)$} \\
\hline Mild & $9(20.5)$ & $43(16.3)$ & $<0.001$ \\
\hline Mild to moderate & $10(22.7)$ & $58(22)$ & 0.002 \\
\hline Moderate to severe & $5(11.4)$ & $43(16.3)$ & $<0.001$ \\
\hline Severe & $4(9.1)$ & $19(7.2)$ & 0.002 \\
\hline Profound & $1(2.3)$ & $9(3.4)$ & 0.011 \\
\hline
\end{tabular}

$\mathrm{HI}=$ hearing impairment; $\mathrm{IQR}=$ interquartile range; $\mathrm{DM}=$ diabetes mellitus; $\mathrm{HbAlc}=$ glycated haemoglobin; $\mathrm{TC}=$ total cholesterol; HDL-C = high-density lipoprotein cholesterol; LDL-C = low-density lipoprotein cholesterol; PCR = protein/creatinine ratio; BMI = body mass index.

Table 5. Analysis of reported symptoms of HI

\begin{tabular}{|c|c|c|c|c|c|}
\hline & Dizziness $(N=120)$ & Vertigo $(N=64)$ & Tinnitus $(N=128)$ & Hearing loss $(N=122)$ & $p$-value \\
\hline Age (years), median (IQR) & $58(50.5-65)$ & $56(47-61.5)$ & $58(50.5-66)$ & $60.5(53-68)$ & 0.03 \\
\hline Duration of DM (years), median (IQR) & $9(4-15.75)$ & $8(5-17)$ & $10.5(5.5-17)$ & $9(5-17)$ & 0.734 \\
\hline \multicolumn{6}{|l|}{ Gender, $n(\%)$} \\
\hline Female & $105(87.5)$ & $56(87.5)$ & $109(85.2)$ & $105(86.1)$ & $<0.001$ \\
\hline Male & $15(12.5)$ & $8(12.5)$ & $19(14.8)$ & $17(13.9)$ & 0.198 \\
\hline \multicolumn{6}{|l|}{ Type of DM, $n(\%)$} \\
\hline T1DM & $8(6.7)$ & $5(7.8)$ & $9(7.0)$ & $4(3.3)$ & 0.455 \\
\hline T2DM & $112(93.3)$ & $59(92.2)$ & $119(93.0)$ & $118(96.7)$ & $<0.001$ \\
\hline Patients with HPT, $n(\%)$ & $105(87.5)$ & $53(82.8)$ & $112(87.5)$ & $113(92.6)$ & $<0.001$ \\
\hline HIV-infected patients, $n(\%)$ & $19(15.8)$ & $10(15.6)$ & $22(17.2)$ & $19(15.6)$ & 0.201 \\
\hline Patients on ART, $n(\%)$ & $13(10.8)$ & $8(12.5)$ & $19(14.8)$ & $16(13.1)$ & 0.194 \\
\hline \multicolumn{6}{|l|}{ Test results, median (IQR) } \\
\hline HbAlc (\%) & $9.7(7.58-11.1)$ & $10.5(8.4-11.3)$ & $9.3(7.6-11.1)$ & $9.2(7.2-10.8)$ & 0.487 \\
\hline $\mathrm{TC}(\mathrm{mmol} / \mathrm{L})$ & $4.5(3.7-5.4)$ & $4.5(3.6-5.2)$ & $4.4(3.5-5.1)$ & $4.6(3.6-5.3)$ & 0.773 \\
\hline Triglyceride $(\mathrm{mmol} / \mathrm{L})$ & $1.58(1.05-2.51)$ & $1.45(0.97-2.18)$ & $1.39(0.99-2.23)$ & $1.59(1.07-2.29)$ & 0.294 \\
\hline HDL-C (mmol/L) & $1.22(1.03-1.5)$ & $1.32(1.11-1.54)$ & $1.16(1.01-1.44)$ & $1.22(1.07-1.46)$ & 0.173 \\
\hline LDL-C (mmol/L) & $2.39(1.73-3.15)$ & $2.4(1.74-3.14)$ & $2.21(1.7-2.99)$ & $2.3(1.66-3.01)$ & 0.838 \\
\hline CD4 (cells/ $\mu \mathrm{L})$ & $\begin{array}{l}559.11(450.31 \text { - } \\
860.45)\end{array}$ & $\begin{array}{l}518(476.67- \\
736.63)\end{array}$ & $\begin{array}{l}519.5(405.31 \text { - } \\
820.29)\end{array}$ & $\begin{array}{l}618(397.95 \text { - } \\
1110.36)\end{array}$ & 0.617 \\
\hline Creatinine $(\mathrm{mmol} / \mathrm{L})$ & $83(67.25-118.75)$ & $74(59.5-107.41)$ & $81(65-123)$ & $93(66-129)$ & 0.243 \\
\hline BMI $\left(\mathrm{kg} / \mathrm{m}^{2}\right)$ & $32(28-39)$ & $31(29-39)$ & $32(28-38)$ & $31(28-38)$ & 0.707 \\
\hline Urinary PCR & $0.03(0.02-0.07)$ & $0.08(0.04-0.22)$ & $0.06(0.02-0.10)$ & $0.03(0.02-0.06)$ & 0.406 \\
\hline \multicolumn{6}{|l|}{ Diabetes-related complications, $n$ (\%) } \\
\hline Non-proliferative retinopathy & $7(5.8)$ & $8(12.5)$ & $13(10.2)$ & $12(9.8)$ & 0.457 \\
\hline Proliferative retinopathy & $2(1.7)$ & $2(3.1)$ & $5(3.9)$ & $3(2.5)$ & 0.572 \\
\hline Sensory neuropathy & $66(55)$ & $35(54.7)$ & $69(53.9)$ & $70(57.4)$ & 0.003 \\
\hline \multicolumn{6}{|l|}{ Antidiabetic therapy, $n(\%)$} \\
\hline Metformin alone & $9(7.5)$ & $7(10.9)$ & $6(4.7)$ & $6(4.9)$ & 0.836 \\
\hline Insulin alone & $62(51.7)$ & $32(50.0)$ & $71(55.5)$ & $70(57.4)$ & $<0.001$ \\
\hline OADs + insulin & $43(35.8)$ & $23(35.9)$ & $47(36.7)$ & $41(33.6)$ & 0.032 \\
\hline
\end{tabular}




\begin{tabular}{|c|c|c|c|c|c|}
\hline & Dizziness $(N=120)$ & Vertigo $(N=64)$ & Tinnitus $(N=128)$ & Hearing loss $(N=122)$ & $p$-value \\
\hline \multicolumn{6}{|c|}{ Patient characteristics, $n(\%)$} \\
\hline \multicolumn{6}{|l|}{ Age (years) } \\
\hline$\leq 50$ & $30(25)$ & $23(35.9)$ & $32(25.0)$ & $23(18.9)$ & 0.485 \\
\hline$>50$ & $90(75)$ & $41(64.1)$ & $96(75.0)$ & $99(81.2)$ & $<0.001$ \\
\hline \multicolumn{6}{|c|}{ Duration of DM (years) } \\
\hline$\leq 10$ & $63(54.8)$ & $36(56.3)$ & $60(46.9)$ & $60(49.2)$ & 0.033 \\
\hline$>10$ & $52(45.2)$ & $22(34.4)$ & $60(46.9)$ & $54(44.3)$ & $<0.001$ \\
\hline \multicolumn{6}{|l|}{$\mathrm{BMI}^{*}\left(\mathrm{~kg} / \mathrm{m}^{2}\right)$} \\
\hline$\leq 25$ & $18(16.8)$ & $8(12.5)$ & $16(12.5)$ & $15(12.3)$ & 0.263 \\
\hline$>25$ & $89(83.2)$ & $45(70.3)$ & $104(81.3)$ & $96(78.7)$ & $<0.001$ \\
\hline \multicolumn{6}{|l|}{$\operatorname{HbAlc}^{*}(\%)$} \\
\hline$\leq 7$ & $22(18.8)$ & $7(10.9)$ & $21(16.4)$ & $23(18.9)$ & 0.025 \\
\hline$>7$ & $95(81.2)$ & $53(82.8)$ & $101(78.9)$ & $94(77.1)$ & $<0.001$ \\
\hline \multicolumn{6}{|l|}{$\mathrm{TC}^{\star}(\mathrm{mmol} / \mathrm{L})$} \\
\hline$\leq 4$ & $36(31.6)$ & $22(34.4)$ & $46(35.9)$ & $38(31.2)$ & 0.038 \\
\hline$>4$ & $78(68.4)$ & $37(57.8)$ & $76(57.8)$ & $72(59.0)$ & $<0.001$ \\
\hline \multicolumn{6}{|c|}{ Triglyceride $^{\star}(\mathrm{mmol} / \mathrm{L})$} \\
\hline$\leq 1.7$ & $67(59.3)$ & $39(60.9)$ & $76(59.4)$ & $62(50.8)$ & 0.007 \\
\hline$>1.7$ & $46(40.7)$ & $20(31.3)$ & $45(35.2)$ & $46(35.7)$ & 0.006 \\
\hline \multicolumn{6}{|c|}{ 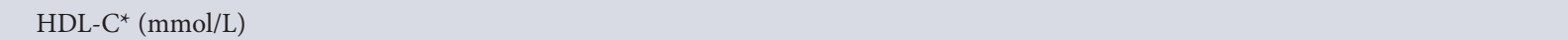 } \\
\hline$\leq 1.2$ & $46(49.5)$ & $16(25.0)$ & $59(46.1)$ & $42(34.4)$ & $<0.001$ \\
\hline$>1.2$ & $47(50.5)$ & $33(51.6)$ & $50(39.1)$ & $53(43.4)$ & 0.162 \\
\hline \multicolumn{6}{|c|}{$\mathrm{LDL}^{-\mathrm{C}^{\star}}(\mathrm{mmol} / \mathrm{L})$} \\
\hline$\leq 1.8$ & $25(34.2)$ & $11(17.2)$ & $31(24.2)$ & $27(22.1)$ & 0.021 \\
\hline$>1.8$ & $48(65.8)$ & $29(45.3)$ & $58(45.3)$ & $45(36.9)$ & 0.022 \\
\hline \multicolumn{6}{|c|}{$\mathrm{GFR}^{\star}\left(\mathrm{mL} / \mathrm{min} / 1.73 \mathrm{~m}^{2}\right)$} \\
\hline$<60$ & $52(45.2)$ & $23(35.9)$ & $49(38.3)$ & $60(49.2)$ & $<0.001$ \\
\hline$\geq 60$ & $63(54.8)$ & $37(57.8)$ & $69(53.9)$ & $54(44.3)$ & 0.015 \\
\hline \multicolumn{6}{|c|}{ Creatinine ${ }^{\star}(\mathrm{mmol} / \mathrm{L})$} \\
\hline$\leq 104$ & $77(67)$ & $45(70.3)$ & $81(63.3)$ & $74(60.7)$ & 0.009 \\
\hline$>104$ & $38(33)$ & $15(23.4)$ & $37(28.9)$ & $40(32.8)$ & 0.005 \\
\hline \multicolumn{6}{|c|}{ Patients reporting symptoms of, $n$ (\%) } \\
\hline Dizziness & $120(100)$ & $40(62.2)$ & $69(53.9)$ & $65(53.3)$ & $<0.001$ \\
\hline Vertigo & $40(25)$ & $64(100)$ & $35(27.3)$ & $31(25.4)$ & 0.001 \\
\hline Tinnitus & $69(57.5)$ & $35(54.7)$ & $128(100)$ & $75(61.5)$ & $<0.001$ \\
\hline Hearing loss & $65(54.2)$ & $31(48.4)$ & $75(58.6)$ & $122(100)$ & $<0.001$ \\
\hline \multicolumn{6}{|c|}{ Ear involvement, $n$ (\%) } \\
\hline Right & $64(53.3)$ & $36(56.3)$ & $64(50.0)$ & $76(62.3)$ & 0.002 \\
\hline Left & $67(55.8)$ & $39(60.9)$ & $67(52.3)$ & $80(65.6)$ & 0.003 \\
\hline Unilateral & $11(9.2)$ & $3(4.7)$ & 15 (11.7) & $16(13.1)$ & 0.025 \\
\hline Bilateral & $60(50.0)$ & $36(56.3)$ & $58(45.3)$ & $70(57.4)$ & 0.012 \\
\hline
\end{tabular}

Idugboe's study, in which mild, moderate and severe were the top HI categories. In the HIV-infected cohort, mild and severe HI were the most frequent categories encountered. A significant proportion of patients across all categories of $\mathrm{HI}$ had a BMI $>25$, highlighting the underlying interaction between obesity, DM and HI.

Approximately half of the PLWD with confirmed HI reported symptoms of dizziness, tinnitus and subjective hearing loss, while a quarter of these patients had vertigo. Significant associations were noted between dizziness, tinnitus and subjective hearing loss and audiological evidence of HI. These associations are important, as they highlight the need for thorough history-taking from PLWD during routine clinic visits. Early detection of $\mathrm{HI}$ can facilitate appropriate audiology referrals together with enhanced lifestyle and therapeutic interventions. No significant association was noted between the mild category and any of the four symptoms. Mild HI is therefore easy to miss, which strengthens the case for annual audiological assessments in PLWD.

We showed that HI was significantly associated with T2DM, HPT, sensory neuropathy, age $>50$ years, a lower GFR and high creatinine levels. A significant proportion of these patients with HI had had DM 


\section{Table 6. Univariate analysis}

\begin{tabular}{|c|c|c|c|c|}
\hline & \multicolumn{2}{|c|}{ HI, $n$} & \multirow[b]{2}{*}{ Unadjusted OR (95\% CI) } & \multirow[b]{2}{*}{$p$-value } \\
\hline & Yes & No & & \\
\hline \multicolumn{5}{|l|}{ Age (years) } \\
\hline$\leq 50$ & 17 & 73 & $8.526(4.67-5.565)$ & $<0.001$ \\
\hline$>50$ & 137 & 69 & & \\
\hline \multicolumn{5}{|l|}{ Gender } \\
\hline Male & 33 & 34 & $0.866(0.502-1.494)$ & 0.606 \\
\hline Female & 121 & 108 & & \\
\hline \multicolumn{5}{|c|}{ Duration of DM (years) } \\
\hline$\leq 10$ & 71 & 90 & $2.113(1.303-3.425)$ & 0.002 \\
\hline$>10$ & 75 & 45 & & \\
\hline \multicolumn{5}{|l|}{ Type of DM } \\
\hline T1DM & 5 & 27 & & $<0.001$ \\
\hline $\mathrm{T} 2 \mathrm{DM}$ & 149 & 115 & & 0.036 \\
\hline \multicolumn{5}{|l|}{ HPT } \\
\hline Yes & 146 & 107 & $5.970(2.662-13.386)$ & $<0.001$ \\
\hline No & 8 & 35 & & \\
\hline \multicolumn{5}{|l|}{ HIV infection } \\
\hline Yes & 22 & 23 & & 0.881 \\
\hline No & 132 & 119 & & 0.412 \\
\hline \multicolumn{5}{|l|}{ ART use } \\
\hline Yes & 21 & 17 & & 0.516 \\
\hline No & 133 & 125 & & 0.618 \\
\hline \multicolumn{5}{|l|}{ HbAlc (\%) } \\
\hline$\leq 7$ & 26 & 25 & $1.075(0.586-1.971)$ & 0.815 \\
\hline$>7$ & 123 & 110 & & \\
\hline \multicolumn{5}{|l|}{$\mathrm{TC}(\mathrm{mmol} / \mathrm{L})$} \\
\hline$>4$ & 54 & 53 & $1.155(0.713-1.873)$ & 0.558 \\
\hline$\leq 4$ & 93 & 79 & & \\
\hline \multicolumn{5}{|l|}{ HDL-C (mmol/L) } \\
\hline$<1.2$ & 53 & 57 & $1.497(0.891-2.516)$ & 0.127 \\
\hline$\geq 1.2$ & 71 & 51 & & \\
\hline \multicolumn{5}{|c|}{ Triglyceride (mmol/L) } \\
\hline$>1.7$ & 86 & 87 & $1.421(0.866-2.332)$ & 0.164 \\
\hline$\leq 1.7$ & 59 & 42 & & \\
\hline \multicolumn{5}{|l|}{ LDL-C (mmol/L) } \\
\hline$>1.8$ & 30 & 28 & $0.948(0.509-1.768)$ & 0.868 \\
\hline$\leq 1.8$ & 63 & 62 & & \\
\hline \multicolumn{5}{|l|}{$\operatorname{BMI}\left(\mathrm{kg} / \mathrm{m}^{2}\right)$} \\
\hline$\leq 25$ & 25 & 27 & $1.24(0.677-2.273)$ & 0.486 \\
\hline$>25$ & 116 & 101 & & \\
\hline \multicolumn{5}{|l|}{ Creatinine $(\mathrm{mmol} / \mathrm{L})$} \\
\hline$>104$ & 60 & 21 & $3.721(2.102-6.585)$ & $<0.001$ \\
\hline$\leq 104$ & 86 & 112 & & \\
\hline \multicolumn{5}{|c|}{$\operatorname{GFR}\left(\mathrm{mL} / \mathrm{min} / 1.73 \mathrm{~m}^{2}\right)$} \\
\hline$\geq 60$ & 64 & 105 & $0.208(0.123-0.354)$ & 0.002 \\
\hline$<60$ & 82 & 28 & & \\
\hline$<15$ & 5 & 1 & & 0.102 \\
\hline $15-29$ & 20 & 4 & & 0.001 \\
\hline $30-44$ & 30 & 13 & & 0.010 \\
\hline $45-59$ & 27 & 10 & & 0.005 \\
\hline Fundoscopy & & & & \\
\hline Non-proliferative & 17 & 11 & $1.478(0.667-3.274)$ & 0.336 \\
\hline Proliferative & 5 & 3 & $1.555(0.365-6.628)$ & 0.551 \\
\hline Sensory neuropathy & & & & \\
\hline Yes & 80 & 56 & $1.66(1.046-2.634)$ & 0.031 \\
\hline No & 74 & 86 & & \\
\hline
\end{tabular}

Continued ... 
Table 6. (continued) Univariate analysis

\begin{tabular}{|c|c|c|c|c|}
\hline & \multicolumn{2}{|c|}{ HI, $n$} & \multirow[b]{2}{*}{ Unadjusted OR (95\% CI) } & \multirow[b]{2}{*}{$p$-value } \\
\hline & Yes & No & & \\
\hline \multicolumn{5}{|l|}{ Antidiabetic therapy } \\
\hline Insulin only & 91 & 70 & $1.486(0.938-2.353)$ & 0.091 \\
\hline OADs only & 7 & 12 & $0.516(0.197-1.349)$ & 0.177 \\
\hline Metformin only & 10 & 7 & $1.339(0.496-3.619)$ & 0.565 \\
\hline OADs plus insulin & 47 & 51 & $0.784(0.483-1.273)$ & 0.325 \\
\hline \multicolumn{5}{|c|}{ Patients reporting symptoms of } \\
\hline Dizziness & 71 & 49 & & 0.045 \\
\hline Vertigo & 39 & 25 & & 0.080 \\
\hline Tinnitus & 73 & 55 & & 0.112 \\
\hline Subjective hearing loss & 86 & 36 & & $<0.001$ \\
\hline
\end{tabular}

\section{Table 7. Multivariate analysis}

\begin{tabular}{|c|c|c|}
\hline & Adjusted OR & $p$-value \\
\hline $\mathrm{TC}$ & $0.284(0.059-1.369)$ & 0.115 \\
\hline Triglyceride & $2.330(0.868-6.257)$ & 0.093 \\
\hline HDL-C & $3.396(1.282-8.996)$ & 0.014 \\
\hline LDL-C & $3.811(0.825-17.598)$ & 0.087 \\
\hline $\mathrm{HbAlc}$ & $2.032(0.697-5.926)$ & 0.194 \\
\hline GFR $>60\left(\mathrm{~mL} / \mathrm{min} / 1.73 \mathrm{~m}^{2}\right)$ & $0.49(0.142-1.690)$ & 0.259 \\
\hline $\mathrm{BMI}>25 \mathrm{~kg} / \mathrm{m}^{2}$ & $0.411(0.109-1.542)$ & 0.187 \\
\hline Creatinine $>104 \mathrm{mmol} / \mathrm{L}$ & $1.064(0.268-4.226)$ & 0.930 \\
\hline Duration of DM & $1.092(0.434-2.749)$ & 0.851 \\
\hline Non-proliferative retinopathy & $1.354(0.256-7.148)$ & 0.721 \\
\hline Proliferative retinopathy & $0.129(0.003-3.03)$ & 0.204 \\
\hline Sensory peripheral neuropathy & $1.296(0.546-3.077)$ & 0.557 \\
\hline HPT & $4.220(0.737-24.176)$ & 0.106 \\
\hline Age $>50$ years & $6.885(2.178-21.764)$ & 0.001 \\
\hline Gender & $0.852(0.303-2.399)$ & 0.762 \\
\hline
\end{tabular}

for $\leq 10$ years. This finding illustrates that $\mathrm{HI}$ occurs early in the course of DM and must be actively sought for by routine history-taking and/ or audiological screening.

Our study, like those of Lerman-Garber et al. ${ }^{[14]}$ and Agrawal et al. ${ }^{[15]}$ showed that PLWD with HI had significantly poorer glycaemic control than those without $\mathrm{HI}$, while studies by Idugboe et al. ${ }^{[28]}$ and Hlayisi et al. ${ }^{[38]}$ in Africa did not find any association between $\mathrm{HI}$ and glycaemic control. ${ }^{[28,38]}$ Our finding sends a strong message emphasising the need for improved overall diabetes control. Optimal glycaemic control remains elusive globally, especially in developing countries, ${ }^{[20]}$ highlighting the need for the introduction of routine audiological screening of PLWD.

Our results revealed an association between $\mathrm{HI}$ and duration of DM, increased age and female gender. In contrast, both Idugboe et al. ${ }^{[28]}$ and Hlayisi et al. ${ }^{[38]}$ showed in their studies that $\mathrm{HI}$ was associated with patient age $<50$ years. Idugboe et al. ${ }^{[28]}$ further showed that there were no associations between HI, duration of DM, glycaemic control or gender, and Hlayisi et al. ${ }^{[38]}$ found that HI was more prevalent in males and positively associated with duration of DM.

Unlike Idugboe et al., ${ }^{[28]}$ who found that PLWD on oral antidiabetics were at increased risk of developing HI, we found that the majority of our patients with HI were on insulin therapy. ${ }^{[28]}$ This is similar to results reported by Bainbridge et al. ${ }^{[12]}$ and Bamanie and Al-Noury. ${ }^{[26]}$
We postulate that patients requiring insulin therapy are those who have uncontrolled DM with suboptimal HBA1c and a resultant increased prevalence of $\mathrm{HI}$.

Multivariate analysis revealed that HI was significantly associated with age $>50$ years and a lower level of HDL-C, a subtype of cholesterol known to have cardioprotective properties. ${ }^{[34,43]}$ Our results and others from Suzuki et al., ${ }^{[35]}$ Bainbridge et al. ${ }^{[12]}$ and Dabrowski et al. ${ }^{[24]}$ have shown that the association between HDL-C and HI must be actively searched for. Patients with HPT and elevated LDL-C were at 4.22 times and 3.81 times increased risk of developing HI, respectively. We also found that HI was more likely in patients with poorer HbAlc, nonproliferative retinopathy and sensory neuropathy.

\section{Study limitations}

A limitation of this study is that all the patients included had DM, and there was no control group. However, the study was designed to detect the prevalence of $\mathrm{HI}$ in PLWD. Future studies comparing HI between PLWD and those without DM are envisaged.

\section{Conclusions}

Our study showed that older patients, females, and those with increased LDL-C and TC, lower HDL-C, suboptimal glucose control, non-proliferative retinopathy, sensory neuropathy and obesity were 
at increased risk of HI. HPT as a comorbidity also increased the likelihood of PLWD developing HI. Implementing intervention strategies targeting the above risk factors in PLWD would include improving physical fitness (known to improve weight, blood pressure, glycaemic control and HDL-C levels) and optimising blood pressure and glycaemic control as set out in diabetes guidelines. These strategies need to be emphasised at all levels of diabetes healthcare.

Symptoms of HI were significantly associated with objective evidence of $\mathrm{HI}$ for all categories except mild. This finding justifies their inclusion in a thorough diabetic history. However, HI cannot be excluded purely on the basis of history, as we found that the mild category had a poor correlation with symptoms. A high level of clinical suspicion is therefore warranted, especially in PLWD with HPT and suboptimal glycaemic and lipid control, and in those with diabetes-related complications.

\section{Declaration. None.}

Acknowledgements. We thank Mr D Singh for his assistance with the statistical analysis required for this article, and Sister L Ndaba and her nursing team at Edendale Hospital for their sterling work with our diabetes patients.

Author contributions. The principal author (SP) made substantial contributions to conception and design of the work, writing of the article and final approval and agreed to be accountable for all aspects of the research. The co-authors (KHN and KM) made contributions to conception of the work, sanctioned the final approval and agreed to be accountable for all aspects of the research.

\section{Funding. None.}

Conflicts of interest. None.

1. Tucci D, Merson MH, Wilson BS. A summary of the literature on global hearing impairment Current status and priorities for action. Otol Neurotol 2010;31(1):31-41. https://doi.org/10.1097/ mao.0b013e3181c0eae

2. Fukushima H, Cureoglu S, Schachem PA, et al. Cochlear changes in patients with type diabetes mellitus. Otolaryngol Head Neck Surg 2005;133(1):100-106. https://doi.org/10.1016/. otohns.2005.02.004

3. Helzner EP, Contrera KJ. Type 2 diabetes and hearing impairment. Curr Diab Rep 2016;16:3. https:// doi.org/10.1007/s11892-015-0696-0

4. Kakarlapudi V, Sawyer R, Staecker H. The effect of diabetes on sensorineural hearing loss. Otol Neurotol 2003;24(3):382-386. https://doi.org/10.1097/00129492-200305000-00006

5. Nent 5. Ramlakhan M, Divij S, Nishi S. Evaluation of hearing loss in patients with type 2 diabetes
Res Med Sci 2016;4(6):2281-2287. https://doi.org/10.18203/2320-6012.ijrms20161800

Res Med Sci 2016;4(6):2281-2287. https://doi.org/10.18203/2320-6012.jjrms20161800
6. Rózańska-Kudelska M, Chodynicki S, Kinalska I, Kowalska I. Hearing loss in patients with diabetes 6. Rózańska-Kudelska M, Chodynicki S, Kinalska I, Ko
mellitus type II. Otolaryngol Pol 2002;56(5):607-610.

7. Rajendran S, Anandhalakshmi, Mythill B, Viswanatha R. Evaluation of the incidence of sensorineural hearing loss in patients with type 2 diabetes mellitus. Int J Biol Med Res 2011;2(4):982-987.

8. Schade DS, Lorenzi GM, Braffett BH, et al. Hearing impairment and type 1 diabetes in the Diabetes Control and Complications Trial/Epidemiology of Diabetes Interventions and Complications (DCCT EDIC) cohort. Diab Care 2018;41(12):2495-2501. https://doi.org/10.2337/dc18-0625

9. Mujica-Mota MA, Patel N, Saliba I. Hearing loss in type 1 diabetes: Are we facing another microvascular disease? A meta-analysis. Int J Pediatr Otorhinolaryngol 2018;113:38-45. https://doi. org/10.1016/j.jijporl.2018.07.005

10. Harner SG. Hearing in adult-onset diabetes mellitus. Otolaryngol Head Neck Surg 1981;89(2):322-327. https://doi.org/10.1177/019459988108900235

11. Schuknecht HF. Pathology of the Ear. 2nd ed. Philadelphia: Lea \& Febiger, 1993:312-314.

12. Bainbridge K, Hoffman HJ, Cowie CC. Diabetes and hearing impairment in the United States Audiometric evidence from the National Health and Nutrition Examination Survey, 1999 to 2004 . Ann Intern Med 2008;149(1):1-10. https://doi.org/10.7326/0003-4819-149-1-200807010-00231

13. Bhaskar NK, Chalihadan S, Vaswani R, Rehaman CP. Clinical and audiometric assessment of hearing loss in diabetes mellitus. Int J Sci Study 2014;2(4):1-16. https://doi.org/10.1007/s12070-018-1566-2

14. Lerman-Garber I, Cuevas-Ramos D, Valdes S, et al. Sensorineural hearing loss - a common finding in early-onset type 2 diabetes mellitus. Endocr Pract 2012;18(4):549-557. https://doi. org/10.4158?EP11389.OR
15. Agrawal Y, Platz EA, Niparko JK. Prevalence of hearing loss and differences by demographic characteristics among US adults: Data from the National Health and Nutrition Examination Survey, 1999 - 2004. Arch Intern Med 2008;168(14):1522-1530. https://doi.org/10.1001/ Survey, $1999-2004$

16. Farooq M, Ahmed A, Mehmood Z, Waheed A. Frequency of sensorineural hearing loss (SNHL) in type 1 and type 2 diabetic patients. Pakistan J Otolaryngol 2013;29:87-89.

17. Okhovat SA, Moaddab MH, Okhovat SH, et al. Evaluation of hearing loss in juvenile insulindependent patients with diabetes mellitus. J Res Med Sci 2011;16(2):179-183.

18. Dadhich S, Jha SG, Sinha V, Samanth TU. A prospective, observational study of the incidence of sensory neural hearing loss in diabetes mellitus patients. Indian J Otol 2018;24(2):80-82. https:// doi.org/10.4103/indianjotol.INDIANJOTOL_135_16

19. Doseman D, Bahniwal RK, Manisha N, Khadilkar MN. Association between type 2 diabetes mellitus and hearing loss among patients in a coastal city of South India. Indian J Otolaryngol Head Neck Surg 2019;71(2):1422-1425. https://doi.org/10.1007/s12070-018-1499-9

20. UK Prospective Diabetes Study (UKPDS) Group. Intensive blood-glucose control with sulphonylureas or insulin compared with conventional treatment and risk of complications in patients with type 2 diabetes (UKPDS 33). Lancet 1998;352(9131):837-853.

21. Ashkezari SJ, Namiranian N, Rahmanian M, et al. Is hearing impairment in diabetic patients correlated to other complications? J Diab Metab Disord 2018;17:173-179. https://doi.org/10.1007/ s40200-018-0357-3

22. Ren $\mathrm{H}$, Wang $\mathrm{Z}$, Mao Z, et al. Hearing loss in type 2 diabetes in association with diabetic neuropathy. Arch Med Res 2018;49(2):631-637. https://doi.org/10.1016/j.arcmed.2018.02.001

23. Taylor IG, Irwin J. Some audiological aspects of diabetes mellitus. J Laryngol Otol 1978;92(2):99113. https://doi.org/10.1017/s0022215100085108

24. Dabrowski M, Nieddzielska GM, Nowakowski A. Impact of different modifiable factors on hearing function in type 1 and type 2 diabetic subjects: A preliminary study. Ann Agricult Environ Med 2013;20(4):773-778.

25. Baiduc RR, Helzner EP. Epidemiology of diabetes and hearing loss. Semin Hear 2019;40(4):281291. https://doi.org/10.1056/s-0039-1697643

26. Bamanie AH, Al-Noury KI. Prevalence of hearing loss among Saudi type 2 diabetic patients. Saudi Med J 2011;32(3):271-274.

27. Ma F, Gómez-Marín O, Lee DJ, Balkany T. Diabetes and hearing impairment in Mexican American adults: A population-based study. J Laryngol Otol 1998;112(9):835-839. https://doi.org/10.1017/ 0022215100141842

28. Idugboe OJ, Kolawole BA, Totyen EL. Hearing threshold level among adult diabetics in southwestern Nigeria. J Otolaryngol Rhinol 2018;4(2):051. https://doi.org/10.23937/2572-4193.1510051

29. Shen FC, Hsieh CJ. Severity of hearing impairment is positively associated with urine albumin excretion rate in patients with type 2 diabetes. J Diabetes Investig 2014;5(6):743-747. https://doi. org/10.1111/jdi.12196

30. Curhan SG, Eavey R, Wang M, Stampfer MJ, Curhan GC. Body mass index, waist circumference, physical activity, and risk of hearing loss in women. Am J Med 2013;126(12):1142-el. https://doi. org/10.1016/j.amjmed.2013.04.026

31. Fransen E, Topsakal V, Hendrickx JJ. Occupational noise, smoking, and a high body mass index are risk factors for age-related hearing impairment, and moderate alcohol consumption is protective: A European population-based multicenter study. J Assoc Res Otolaryngol 2008;9(3):264-276. https://doi.org/10.1007/s10162-008-0123-1

32. Swaminathan A, Sambandam R, Bhaskaran M. Evaluation of the auditory effects of hyperlipidaemia and diabetes mellitus by using audiometry. J Clin Diagn Res 2011;5(8):1528-1532. https://doi.org/ JCDR/2011/1783

33. Evans MB, Tonini R, Shope CD, et al. Dyslipidemia and auditory function. Otol Neurotol 2006;27(5):609614. https://doi.org/10.1097/01.mao.0000226286.19295.34

34. Nagao M, Nakajima H, Toh R, Hirata KI, Ishida T. Cardioprotective effects of high-density lipoprotein beyond its anti-atherogenic action. J Atheroscler Thromb 2018;25(10):985-993. https:// doi.org/10.5551/jat.RV17025

35. Suzuki K, Kaneko M, Murai K. Influence of serum lipids on auditory function. Laryngoscope 2000;110(10):1736-1738. https://doi.org/10.1097/00005537-200010000-00033

36. Dalton DS, Cruickshank KJ, Klein R, Klein BE, Willey TL. Association between NIDDM and hearing loss. Diabetes Care 1998;21(9):1540-1544. https://doi.org/10.2337/diacare.21.9.1540

37. Sindhusake D, Mitchell P, Smith W, et al. Validation of self-reported hearing loss: The Blue Mountains Hearing Study. Int J Epidemiol 2001;30(6):1371-1378. https://doi.org/10.1093/ ije/30.6.1371

38. Hlayisi VG, Peterson L, Ramma L. High prevalence of disabling hearing loss in young to middleaged adults with diabetes. Int J Diab Dev Countries 2018;39:148-153. https://doi.org/10.1007/ s13410-018-0655-9

39. Bener A, Al-Hamaq A, Abdulhadi K, Salahaldin AH, Gansan L. Interaction between diabetes mellitus and hypertension on the risk of hearing loss in a highly endogamous population. Diabetes Metab Syndr 2017;11(1):545-551. https://doi.org/10.1016/j.dsx.2016.09.004

40. SEMDSA Type 2 Diabetes Guidelines Expert Committee. SEMDSA 2017 guidelines for the management of type 2 diabetes mellitus. J Endocr Metab Diabetes S Afr 2017;22(1 (Suppl 1)):S1S196. https://doi.org/10.13140/RG.2.2.29645.90083

41. Kidney Disease: Improving Global Outcomes (KDIGO) CKD Work Group. KDIGO 2012 clinical practice guideline for the evaluation and management of chronic kidney disease. Kidney Int Suppl 2013;3(1):1-150.

42. Souza SJ, Luzia LA, Santos SR, Rondó PHC. Lipid profile of HIV-infected patients in relation to antiretroviral therapy: A review. Rev Assoc Med Bras 2013;59(2):186-198. https://doi.org/10.1016/j. ramb.2012.11.003

43. Van Linthout S, Frias M, Singh N, de Geest B. Therapeutic potential of HDL in cardioprotection and tissue repair. Handb Exp Pharmacol 2015;224:527-565. https://doi.org/10.1007/978-3-31909665-0_17

Accepted 28 June 2021. 